\title{
SOUTHERN REFERENCE STAR PROGRAM: PROGRESS REPORT
}

\author{
J. L. SCHOMBERT
}

U.S. Naval Observatory, Washington, D.C., U.S.A.

The observational programs for the Southern Reference Star Program are rapidly drawing to a close. Observations have been completed, except for a few at San Juan and a small percentage of zone $-52^{\circ}$ to $-64^{\circ}$ at the Cape. The Cape zone $-64^{\circ}$ to $-90^{\circ}$ has been cancelled. The current state of the observations is shown in Table I.

Final results have now been received from Abbadia, Bordeaux, Tokyo, USNO 6-in. and Cape (zone $-40^{\circ}$ to $-52^{\circ}$ ). These results are in machine readable form and preliminary investigations have begun. Results from several other observatories are expected in a short time.

\section{TABLE I}

Status of the SRS programme - 1 June 1973

\begin{tabular}{|c|c|c|c|c|c|c|}
\hline \multirow[t]{2}{*}{ Observatory } & \multirow[t]{2}{*}{ Zone } & \multirow[t]{2}{*}{ Commitment } & \multirow{2}{*}{$\begin{array}{l}\text { Date } \\
\text { started }\end{array}$} & \multicolumn{2}{|c|}{ Completed } & \multirow{2}{*}{$\begin{array}{l}\text { Final results } \\
\text { received or } \\
\text { expected }\end{array}$} \\
\hline & & & & Obs'ns & Red'ns & \\
\hline Abbadia & $+5^{\circ}$ to $-15^{\circ}$ & $1560 \times 4$ & 62.3 & $100 \%$ & $100 \%$ & 1968.8 \\
\hline Bordeaux & +5 to -15 & $1560 \times 4$ & 62.5 & 100 & 100 & 1970.5 \\
\hline Bucharest & +5 to -10 & $1176 \times 4$ & 62.6 & 100 & 97 & 1975.0 \\
\hline Nicolaiev & 0 to -20 & $5984 \times 2$ & 64.3 & 100 & 98 & 1974.0 \\
\hline San Fernando & -10 to -30 & $3709 \times 4$ & 63.3 & 100 & 100 & 1973.5 \\
\hline Tokyo & -10 to -30 & $3560 \times 4$ & 63.3 & 100 & 100 & 1972.7 \\
\hline USNO 6-in. & +5 to -30 & $\begin{array}{l}8706 \times 2 \\
1233 \times 4\end{array}$ & 66.5 & 100 & 100 & 1973.3 \\
\hline $\begin{array}{l}\text { Bergedorf } \\
\text { (Bickley) }\end{array}$ & +5 to -90 & $20495 \times 4$ & 67.9 & 100 & 95 & 1974.5 \\
\hline Cape & $\begin{array}{l}-30 \text { to }-40 \\
-40 \text { to }-52 \\
-52 \text { to }-64 \\
-64 \text { to }-90\end{array}$ & $10082 \times 4$ & 61.3 & $\begin{array}{r}100 \\
100 \\
80 \\
-\end{array}$ & $\begin{array}{r}97 \\
100 \\
75 \\
-\end{array}$ & $\begin{array}{l}1974.3 \\
1966.0 \\
1974.5 \\
-\end{array}$ \\
\hline San Juan & -40 to -90 & $7190 \times 2$ & 69.5 & 99.4 & 100 & 1974.3 \\
\hline Santiago - & -25 to -47 & $5992 \times 4$ & 63.1 & 100 & 60 & 1974.0 \\
\hline Pulkovo & -47 to -90 & $5504 \times 4$ & & 100 & 75 & 1975.0 \\
\hline $\begin{array}{l}\text { USNO 7-in. } \\
\text { (El Leoncito) }\end{array}$ & $\begin{array}{l}+5 \text { to }-20 \\
-20 \text { to }-75 \\
-75 \text { to }-75 \mathrm{SP}\end{array}$ & $\begin{array}{r}7683 \times 2 \\
12121 \times 4 \\
1382 \times 4\end{array}$ & 68.7 & 100 & 71 & 1975.0 \\
\hline
\end{tabular}

